\title{
AN EFFICIENT WEIGHT OPTIMIZATION ALGORITHM FOR IMAGE REPRESENTATION USING NONORTHOGONAL BASIS VECTORS
}

\author{
Yuk-Hee Chan and Wan-Chi Siu
}

\author{
Department of Electronic Engineering \\ The Hong Kong Polytechnic University \\ Hung Hom, Kowloon, Hong Kong
}

\begin{abstract}
Though image-coding techniques that employ subsets of nonorthogonal basis images chosen from two or more transform domains have been shown consistently to yield higher image quality than those based on one transform for a fixed compression ratio, they have not been widely employed due to their very high computational complicity of existing realization approaches. This paper presents a new realization approach for mixed-transform image representation. Computational complexity can be greatly reduced compared with existing approaches.
\end{abstract}

\section{INTRODUCTION}

Recently, mixed-transform coding techniques have been proposed to represent a signal from subsets of basis vectors selected from multiple transform domain[1-4]. These coders have been shown to yield higher image quality compared with those based on a single transform. However, they have not been widely employed because of their very high computational complexity. This paper presents a new realization approach for mixed-transform image representation. The approach greatly reduces the computational complexity compared with existing approaches.

\section{ALGORITHM}

In block-based transform image coding, a block of image can be generally represented as $\mathbf{x}=\mathbf{T} \mathbf{c}$, where $\mathbf{x}$ and c are respectively the lexicographically ordered original image and its corresponding transform coefficient vector, and $\mathbf{T}$ is the corresponding $2 \mathrm{D}$ transformation operator. Note that the $\mathrm{i}^{\text {th }}$ column of $\mathbf{T}$ is actually the lexicographically ordered basis vector associated with the $\mathrm{i}^{\text {th }}$ transform coefficient. Obviously, if different unitary transforms, say $\mathbf{T}_{\mathbf{a}}$ and $\mathbf{T}_{\mathbf{b}}$, are exploited, their

This work was supported by POLYU Grant 0351.504.A3.420. corresponding coefficient vectors will be different and we have $\mathbf{x}=\mathbf{T}_{\mathbf{a}} \mathbf{c}_{\mathbf{a}}=\mathbf{T}_{\mathbf{b}} \mathbf{c}_{\mathbf{b}}$.

Consider a mixed-transform image coder which selects dominant components in the transform domains of these two transforms. The image $\mathbf{x}$ is encoded with $n_{a}$ basis vectors of $\mathbf{T}_{\mathbf{a}}$ and $n_{b}$ basis vectors of $\mathbf{T}_{\mathbf{b}}$. Then the reconstructed version of $\mathbf{x}$, say $\hat{\mathbf{x}}$, can be represented as

$$
\hat{\mathbf{x}}=\mathbf{T}_{\mathbf{a}} \mathbf{u}+\mathbf{T}_{\mathbf{b}} \mathbf{v}
$$

where $\mathbf{u}$ and $\mathbf{v}$ are lexicographically ordered vectors whose elements equal to the weights of selected $\mathbf{T}_{\mathbf{a}}$ and $\mathbf{T}_{\mathbf{b}}$ basis vectors respectively and are zero otherwise. In the transform domain of $\mathbf{T}_{\mathbf{a}}$, it can be represented as

$$
\mathbf{T}_{\mathbf{a}}^{-1} \hat{\mathbf{x}}=\mathbf{u}+\mathbf{T}_{\mathbf{a}}^{-1} \mathbf{T}_{\mathbf{b}} \mathbf{v}
$$

In non-matrix form, we have

$$
\begin{aligned}
\hat{c}_{a, i} & =u_{i}+\sum_{j \in \Lambda_{b}} \alpha_{i j} v_{j} & & \text { for } i \in \Lambda_{a} \\
\hat{c}_{a, i} & =\sum_{j \in \Lambda_{b}} \alpha_{i j} v_{j} & & \text { for } i \notin \Lambda_{a}
\end{aligned}
$$

where $\hat{c}_{a, i}, u_{i}, v_{i}$ are the $\mathrm{i}^{\text {th }}$ elements of $\mathbf{T}_{\mathbf{a}}^{\mathbf{- 1}} \hat{\mathbf{x}}, \mathbf{u}$ and $\mathbf{v}$ respectively, and, $\alpha_{i j}$ is the $\mathrm{i}^{\text {th }}$ element of the $\mathrm{j}^{\text {th }}$ column of $\mathbf{T}_{\mathbf{a}}^{-1} \mathbf{T}_{\mathbf{b}}$. Here, $\Lambda_{a}$ is the set of indices whose associated basis vectors of $\mathbf{T}_{\mathbf{a}}$ are selected and $\Lambda_{b}$ is the set of indices whose associated basis vectors of $\mathbf{T}_{\mathbf{b}}$ are selected.

Our proposed approach finds the best representation of a given image by repeatedly carrying out the following 2 steps until the reduction of the distortion is less than a predefined threshold or a particular criterion is achieved: (i) select a new basis vector via a marginal analysis and update $\Lambda_{a}$ and $\Lambda_{b}$ accordingly, and (ii) determine the weights of the selected basis vectors for the updated $\Lambda_{a}$ and $\Lambda_{b}$. The initial condition 
is $\Lambda_{a}=\Lambda_{b}=\{\}$. In the following, we will describe the 2 steps in detail.

A. Determine the weights of selected basis vectors:

If minimum distortion criterion is exploited, the weights of the selected basis vectors should be determined in a way that the reconstructed $\hat{\mathbf{x}}$ minimizes the distortion $d=\|\mathbf{x}-\hat{\mathbf{x}}\|^{2}$. As $\mathbf{T}_{\mathbf{a}}$ and $\mathbf{T}_{\mathbf{b}}$ are unitary, we have

$$
d=\left\|\mathbf{T}_{\mathbf{a}}^{-\mathbf{1}}(\mathbf{x}-\hat{\mathbf{x}})\right\|^{2}=\sum_{i=0}^{N-1}\left(c_{a, i}-\hat{c}_{a, i}\right)^{2}
$$

where $N$ is the dimension of $\mathbf{x}$ and $c_{a, i}$ is the $\mathrm{i}^{\text {th }}$ element of $\mathbf{c}_{\mathbf{a}}$. The minimum distortion can be achieved by separately minimizing each component in the transform domain of $\mathbf{T}_{\mathbf{a}}$.

Obviously, for given $\Lambda_{a}$ and $\Lambda_{b}$, we can always make $\hat{c}_{a, i}=c_{a, i}$ to reduce the distortion of this component to zero by adjusting $u_{i}$ if $i \in \Lambda_{a}$. Hence, in order to minimize the distortion $d$, all we need to do is to minimize

$$
E=\sum_{i \notin \Lambda_{a}}\left(c_{a, i}-\hat{c}_{a, i}\right)^{2}=\sum_{i \notin \Lambda_{a}}\left(c_{a, i}-\sum_{j \in \Lambda_{b}} \alpha_{i j} v_{j}\right)^{2}
$$

To simplify the formulation of the algorithm, we can rewrite eqn. (5) in matrix form as

$$
E=\|\overrightarrow{\mathbf{c}}-\mathbf{A} \overrightarrow{\mathbf{v}}\|^{2}
$$

where $\overrightarrow{\mathbf{c}}$ and $\overrightarrow{\mathbf{v}}$ are the vectors whose elements are $c_{a, i}$ 's sorted according to $i \notin \Lambda_{a}$ and $v_{i}$ 's sorted according to $i \in \Lambda_{b}$ respectively, and $\mathbf{A}$ is the corresponding matrix composed of $\alpha_{i j}$ 's. By minimizing $E$ with respect to $\overrightarrow{\mathbf{v}}$, we have

$$
\overrightarrow{\mathbf{v}}=\left(\mathbf{A}^{\mathbf{t}} \mathbf{A}\right)^{-\mathbf{1}} \mathbf{A}^{\mathbf{t}} \overrightarrow{\mathbf{c}}
$$

Note that $\mathbf{A}$ is a submatrix of $\mathbf{T}_{\mathbf{a}}^{-\mathbf{1}} \mathbf{T}_{\mathbf{b}}$. As both $\mathbf{T}_{\mathbf{a}}$ and $\mathbf{T}_{\mathbf{b}}$ are orthogonal transform kernels, matrix $\mathbf{A}^{\mathbf{t}} \mathbf{A}$ is of full rank and its inverse exists. Also note the matrix $\mathbf{A}^{\mathbf{t}} \mathbf{A}$ is of size $n_{b} \times n_{b}$. One can always make $n_{a} \geq n_{b}$ as it is free to pick any particular transform as $\mathbf{T}_{\mathbf{a}}$. That implies $n_{b} \leq n / 2$, where $n=n_{a}+n_{b}<N$ is the total basis vectors currently selected. In typical block-based transform coding, we usually have $N=64$. Besides, our approach starts with small $n$. That makes the size of $\mathbf{A}^{\mathbf{t}} \mathbf{A}$ small and hence the direct computation of $\left(\mathbf{A}^{\mathbf{t}} \mathbf{A}\right)^{-1}$ practical.
After obtaining $v_{j}$ 's, the weights of the selected $\mathbf{T}_{\mathbf{a}}$ basis vectors can be determined with $u_{i}=c_{a, i}-$ $\sum_{j \in \Lambda_{b}} \alpha_{i j} v_{j}$ for $i \in \Lambda_{a}$.

\section{B. Select a new basis vector:}

Suppose we have obtained the optimal set of $u_{i}$ 's and $v_{i}$ 's for a given combination of $\Lambda_{a}$ and $\Lambda_{b}$ and want to include one more basis vector. Without loss of generality, we assume the newly included basis vector comes from the transform domain of $\mathbf{T}_{\mathbf{a}}$ and its index is $m$. Then, based on eqns. (3) and the analysis we have made, the guaranteed reduction in distortion is given as

$$
\Delta E_{a, m}=\left(c_{a, m}-\sum_{j \in \Lambda_{b}} \alpha_{m j} v_{j}\right)^{2}
$$

One can determine the best candidate among those not yet selected basis vectors of $\mathbf{T}_{\mathbf{a}}$ by selecting the $m$ whose $\Delta E_{a, m}$ is maximum. In order to reduce the complexity, one can just search the $m$ which maximizes

$$
\Delta E_{a, m}^{\prime}=\left|c_{a, m}-\sum_{j \in \Lambda_{b}} \alpha_{m j} v_{j}\right|
$$

as the same candidate will be picked. By making use of the same approach, we have the best candidate among those not yet selected basis vectors of $\mathbf{T}_{\mathbf{b}}$. By comparing their guaranteed reductions in distortion, one can determine which one should be added.

\section{COMPUTATIONAL COMPLEXITY}

First of all, one has to carry out two transforms to compute $\mathbf{c}_{\mathbf{a}}$ and $\mathbf{c}_{\mathbf{b}}$. Since transforms which have fast realization algorithms are generally exploited, this overhead can be of an order of $N \log _{2} N$ multiplications.

At a particular stage, assume that we have already had $n_{a} \mathbf{T}_{\mathbf{a}}$ and $n_{b} \mathbf{T}_{\mathbf{b}}$ coefficients. Then $n_{b}\left(N-n_{a}\right)+$ $n_{a}\left(N-n_{b}\right)$ multiplications and $n_{b}\left(N-n_{a}\right)+n_{a}\left(N-n_{b}\right)$ additions are required to search the best basis vector to be added. After this, one has to determine the weights of the selected basis vectors. To compute $v_{j}$ 's, if $\left(\mathbf{A}^{\mathbf{t}} \mathbf{A}\right)^{-\mathbf{1}} \mathbf{A}^{\mathbf{t}}$ is precomputed, roughly $n_{b}\left(N-n_{a}\right)$ multiplications and $n_{b}\left(N-n_{a}\right)$ additions will be required. The exact number of operations required depends on whether the newly added basis vector is a basis vector of $\mathbf{T}_{\mathbf{a}}$ or $\mathbf{T}_{\mathbf{b}}$. As for computing $u_{i}$ 's, about $n_{a} n_{b}$ multiplications and $n_{a} n_{b}$ additions are required. In summary, for each iteration, the number of multiplications 
required is $n_{b}\left(N-n_{a}\right)+n_{a}\left(N-n_{b}\right)+n_{b}\left(N-n_{a}\right)+$ $n_{a} n_{b}=N n+N n_{b}-2 n_{a} n_{b} \leq 3 n N / 2$.

Suppose $M$ is the total number of basis vectors selected at the end. The total computational complexity in terms of number of multiplications is then bounded by $\sum_{n=0}^{M-1} 3 n N / 2<3 N M^{2} / 4$. The total number of additions required is also bounded by this figure.

Like the existing mixed-transform coding algorithm [4], the proposed approach iteratively builds up a more accurate representation by including additional basis vectors one at a time. An iterative approach developed with a gradient descendent algorithm is applied in [4] to compute the weights of the selected vectors. It requires approximately $2 n N$ multiplications for each iteration and takes time to converge. However, ours for the same function is non-iterative and takes only less than $n N / 2$ multiplications totally.

\section{SIMULATIONS}

The proposed mixed-transform algorithm was verified through simulations with the discrete cosine transform (DCT) and Haar transform(DHT). Similar performance like those reported in [4] were obtained. Figure 1 shows the coding performance of various image coding schemes in coding "Lenna" which is of size $512 \times 512$. The parameter $\eta$ is defined as the total number of transform coefficients retained to reconstruct the output image divided by the image size. It is obvious that the one using mixed transform provides a better Peak-to-peak Signal Noise Ratio (PPSNR) especially when the value of $\eta$ is high.

Figure 2 shows the simulation results of coding another testing image "Baboon". The original image is of size $512 \times 512$. It was respectively transformed to the DCT, DHT and mixed transform domains. Only $20 \%$ of the most significant $512 \times 512$ transform coefficients were used to reconstruct the output image in each case. Figures 2(b)-(d) respectively show some details of the reconstructed images. They are enlarged for comparison. One can see the superiority of the scheme using mixed transform by examining the details around the eyebrow in the figures.

\section{CONCLUSION}

In this paper, a simple and efficient mixed-transform coding algorithm is presented. This algorithm provides an excellent coding performance and greatly reduces the computational complexity compared to existing re-

\section{PPSNR vs. No. of Retained coefs}

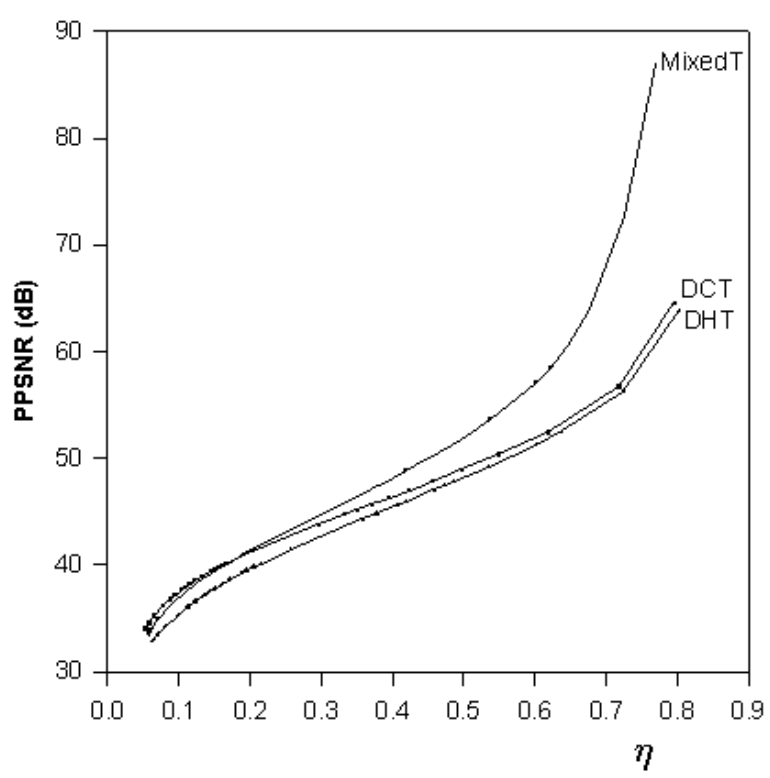

Figure 1: Performance of various coding schemes

alization approaches, which makes mixed-transform image coding practical.

\section{REFERENCES}

[1] W.B. Mikhael and A. Spanias, "Accurate representation of time varying signals using mixed transforms with applications to speech," IEEE Trans. on Circuits and Systems, Vol.36, No.2, pp.329-331, Feb, 1989.

[2] W. B. Mikhael and A. Ramaswamy, "Application of multitransforms for lossy image representation," IEEE trans. on Circuits and Systems-II, Vol.41, No.6, pp.431-434, Jun 1994.

[3] W. B. Mikhael and A. Ramaswamy, "Resolving images in multiple transform domains with applications," Digital Signal Processing: A review journal, Vol.5, No.2, pp.81-90, Apr 1995.

[4] W. B. Mikhael and A. P. Berg, "Image representation using nonorthogonal basis images with adaptive weight optimization," IEEE Signal Processing Letters, Vol.3, No.6, pp.165-167, Jun 1996. 


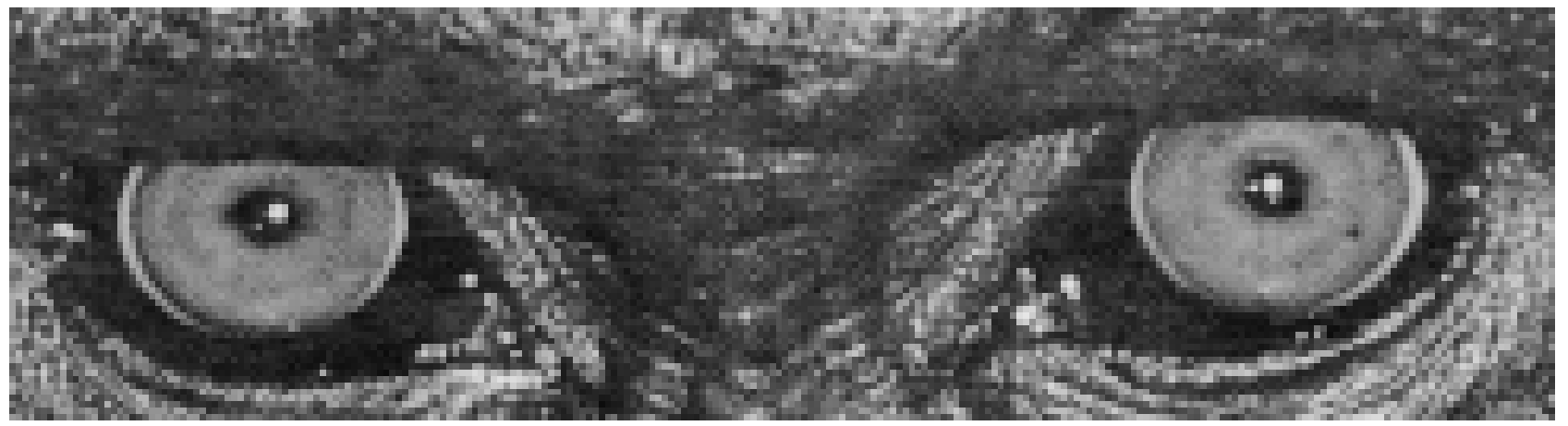

(a)

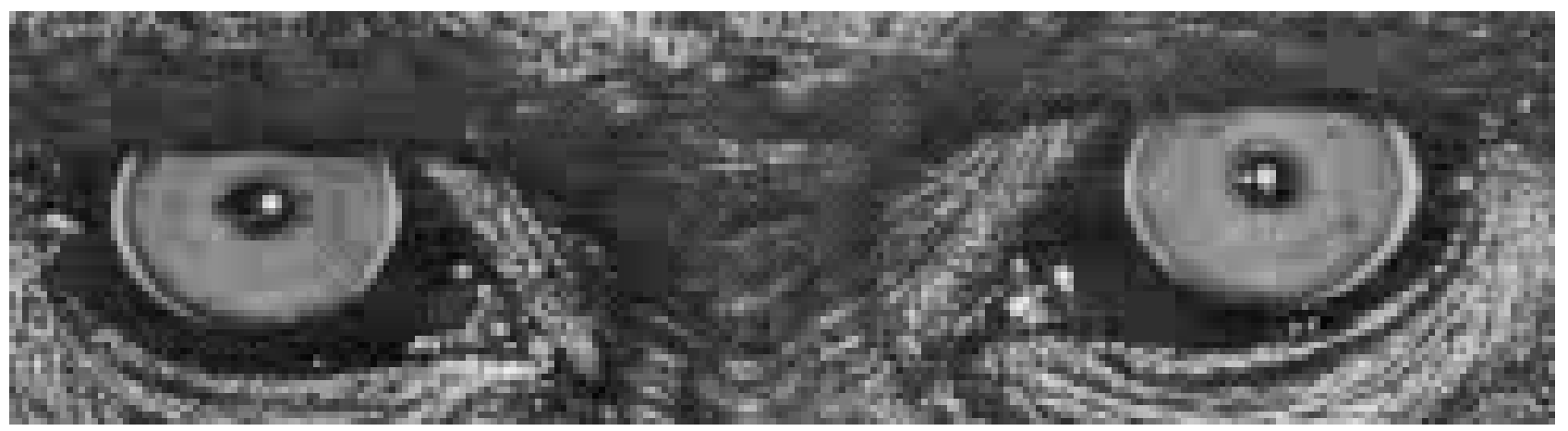

(b)

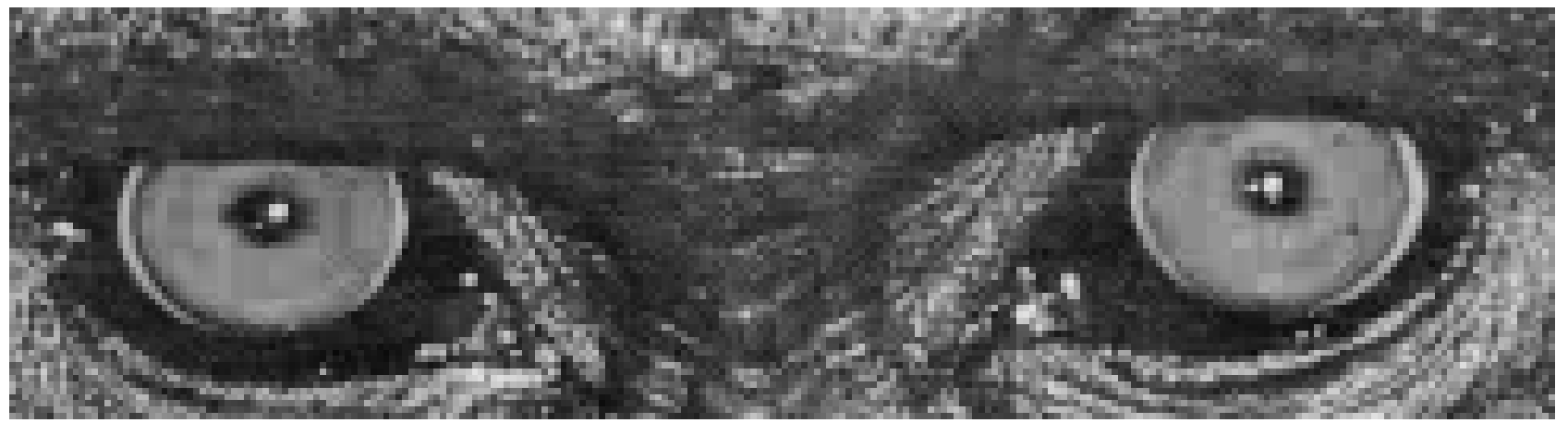

(c)

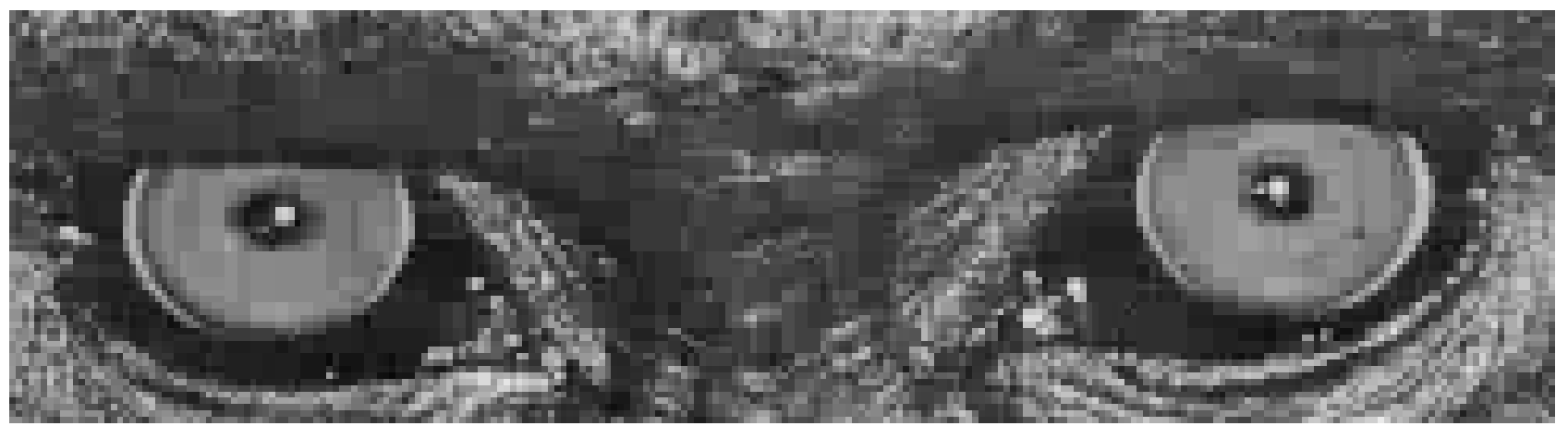

(d)

Figure 2: Enlarged "Baboon" and its encoded results $(\eta=0.2)$ : (a). Original (b). DCT-encoded result (c). Mixedtransform-encoded result and (d). DHT-encoded result 